\title{
Revisiones de precios en los contratos de las Corporaciones locales
}

\author{
$352.712(46)$
}

por

MARIA-ANTONIA ANGULO GONZALEZ

Abogado. Técnico del Excmo. Ayuntamiento de Madrid.

SUMARIO: I. PLANTEAMIENTO DEL PROBLEMA.-II. SOLUCIONES EN DEReCHO PRIVAdO: 1. Revisión ante los Tribunales. 2. Cuáusulas estabilizadoras. 3. Reglamento hipotecario.-III. SOLUCIONES EN DERECHO AD. ministrativo. eVolucion legislativa: 1. Pliego de condiciones de 13 de marzo de 1903. 2. Ley de 17 Julio de 1945. 3. Reglamento de Contratación de las Corporaciones locales. 4. Reglamento de Servicios de las Corporaciones locales. 5. Decretos de 13 de enero y 25 de febrero de 1955. 6. Ley de Con. tratos del Estado. 7. Decreto-ley 2/1964, de 4 de FEbrero.-IV. SiTUACIONES POSIBLES DE LOS CONTRATOS DE ENTES LOCALES: 1. Contratos SIN cláusula de revisión de precios: A) Contratos en general. B) Concesiones. 2. Contratos con cláusula de revisión de precios: A) Contratos de obras. B) Contratos de servicios y suministros.-V. CRITICA DEL SISTEMA.-VI. CON. CLUSIONES PRACTICAS.

\section{PLANTEAMIENTO DEL PROBLEMA}

La forma general de los contratos consiste en obligarse una parte a realizar una prestación, de dar o de hacer, a cambio de una cantidad de dinero. Esta cantidad se presume equivalente a la prestación recibida a cambio, según el principio general de equivalencia de las prestaciones. Sin embargo, en estas obligaciones de pago en dinero, cuando el cumplimiento de la prestación o del pago queda diferido en el tiempo, resulta que la devaluación del dinero hace que una de las partes contratantes quede perjudicada al recibir una cantidad de dinero, que si bien 
es nominalmente la misma que se pactó, tiene un poder adquisitivo inferior al previsto.

El ordenamiento jurídico español, igual que todos los que han tenido su elaboración definitiva a partir de la codificación del siglo XIX, parte de la base económica de la estabilidad de la moneda; de la invariabilidad en el tiempo de su poder adquisitivo. Por ello ordena que lo convenido en un momento determinado debe cumplirse exactamente aunque pasen varios años. Se considera que el encarecimiento de los materiales y de los servicios es un riesgo que deben correr las partes contratantes.

Desgraciadamente, la base económica y social en que descansa ese ordenamiento jurídico ha cambiado en lo que llevamos de siglo. El proceso inflacionista se ha hecho crónico y el poder adquisitivo de todas las monedas cada vez es más reducido. Sin embargo, la legislación no ha cambiado al mismo ritmo, y en los contratos de pago en dinero de ejecución diferida, siempre habrá un perjudicado por esta discrepancia entre Derecho y realidad. Unas veces será el que realice unas prestaciones cuyo pago se aplace, y otras veces será el que tenga que realizarlas al precio que se calculó tiempo atrás. En ambos casos el valor adquisitivo del dinero que recibe como pago será inferior al empleado para realizar su prestación.

\section{SOLUCIONES EN DERECHO PRIVADO}

\section{Revisión ante los Tribunales.}

En primer lugar, la parte contratante perjudicada puede solicitar, amparándose en la cláusula rebus sic stantibus que se entiende implícita en todos los contratos, la resolución o modificación del mismo, fijando el precio que resulte de la nueva situación del mercado. Sin embargo, ¿cómo invocar el cambio de las circunstancias cuando en el momento en que se contrató ya se sabía con certeza que en el transcurso de varios años habría subido el coste de los elementos de la obra o servicio contratado? Se trata de una circunstancia totalmente previsible, que debió tomarse en cuenta al formalizar el contrato. $Y$ por ello, salvo 
casos especiales, no se admite la modificación o resolución del contrato como consecuencia de la cláusula rebus sic stantibus.

\section{Cláusulas estabilizadoras.}

Ante la dificultad de lograr ante los Tribunales, en vista de lo anterior, la modificación de los contratos, se introdujo en la práctica la costumbre de insertar en los que tuviesen una cierta importancia y duración alguna de las llamadas cláusulas estabilizadoras, mediante cuya aplicación se determinaba a posteriori la valoración de las prestaciones contratadas, sin que esto supusiese modificación del contrato, sino sólo cumplimiento del mismo. Se dejaba la cuantía de la prestación no determinada, sino determinable, en virtud de ciertas circunstancias. Así, por ejemplo, el precio que se hace depender del índice del coste de la vida, del valor del oro, de una moneda extranjera o de cualquier otro dato.

\section{Reglamento hipotecario.}

Este tipo de cláusulas ha logrado incluso su reconocimiento expreso en una norma legal, con una regulación detallada de su contenido. Así ha sucedido con los préstamos hipotecarios, para los que el apartado 3. del artículo 219 del Reglamento hipotecario de 14 de febrero de 1947 admite y regula con detalle la posibilidad de las que llama «cláusulas de estabilización de valor».

\section{SOLUCIONES EN DERECHO ADMINISTRATIVO. EVOLUCION LEGISLATIVA}

1. Pliego de condiciones de 13 de marzo de 1903.

El pliego de condiciones de 13 de marzo de 1903 seguía el criterio rígido de sujeción estricta al contrato (contractus lex), sin admitir variación alguna de sus términos más que por una serie de causas de la categoría de fuerza mayor, entre las que no estaba comprendida la alteración de los precios del mercado (artículos 32, 42 y 43 ). 


\section{LEY DE 17 DE JULIO DE 1945.}

Sin embargo, por Ley de la Jefatura del Estado de 17 de julio de 1945 se estableció con carácter general la revisión de precios, con una gran generosidad para el contratista. Se trata de una revisión ex lege, independiente de las posibles revisiones ex contractu, pues su artículo 11 establece que «no serán de aplicación sus preceptos a aquellos contratos en que estén previstos los casos de revisión o forma de llevarlos a cabo, en los que se estará a lo especialmente pactado en cada uno de ellos». Hay que destacar que esta revisión ex lege, como señala acertadamente Gaspar Ariño Ortiz, no trata de nivelar los precios del contrato con los reales, sino con los fijados por disposiciones de la autoridad pública. Es decir, que se trata de una consecuencia lógica de un acto propio (teoría del factum principis, según la cual el Estado tiene que atenerse y ser consecuente con las órdenes que ha dictado él mismo. $O$ sea, que si ordena el cambio de un precio determinado tendrá el mismo Estado que aplicarlo en sus propios contratos).

3. Reglamento de Contratación de las Corporaciones locales.

En el mismo sentido, en la esfera local, el Reglamento de Contratación de las Corporaciones locales de 9 de enero de 1953 establece lo siguiente: «En los contratos en que intervengan las Corporaciones locales se entenderán siempre convenidos a riesgo y ventura para el contratista, sin que éste pueda solicitar alteración del precio o indemnización, excepto por alguna de las siguientes causas:

a) Incendios originados por la electricidad atmosférica.

b) Daños producidos por terremotos.

c) Daños derivados de movimientos del terreno en que se estén construyendo las obras.

d) Destrozos ocasionados violentamente a mano armada en tiempo de guerra, o por sediciones populares o robos tumultuo$\operatorname{sos} ; \mathbf{y}$

e) Aumentos que excedan del 10 por 100 del precio de los materiales o jornales que de hecho viniere satisfaciendo el con- 
tratista, cuando fueren establecidos por precepto obligatorio y no existiere demora imputable a aquél en relación con los plazos señalados por el pliego de condiciones».

\section{Reglamento de Servicios de las Corporaciones locales.}

En cambio, el Reglamento de Servicios de las Corporaciones locales, de 17 de junio de 1955, establece en su articulo 127-2 que la Corporación concedente deberá: «2. Mantener el equilibrio financiero de la concesión, para lo cual:

a) Compensará económicamente al concesionario por razón de las modificaciones que le ordenare introducir en el servicio y que incrementaren los costos o disminuyeren la retribución;

b) Revisará las tarifas y subvención cuando, aun sin mediar modificaciones en el servicio, circunstancias sobrevenidas e imprevisibles determinaren, en cualquier sentido, la ruptura de la economía de la concesión».

En el mismo sentido, el artículo 126-2-b), al hablar de la retribución económica del concesionario, establece que su «equilibrio, a tenor de las bases que hubieren servido para su otorgamiento, deberá mantenerse en todo caso y en función de la necesaria amortización, durante el plazo de concesión, del coste de establecimiento del servicio que hubiere satisfecho, así como de los gastos de explotación y normal beneficio industrial».

\section{Decretos de 13 de ENERo Y 25 DE FEBRero de 1955.}

Sin embargo, en la esfera de la Administración central, y por Decreto de 13 de enero de 1955, se suspendió la revisión de precios de los contratos celebrados por el Estado que se estaban realizando al amparo de la Ley de 1945.

Paralelamente, para la Administración local, se suspendió el apartado e) del artículo 57 del Reglamento de Contratación, por Decreto de 25 de febrero de 1957.

A pesar de ello, poco tiempo después se autorizó la modificación «por una sola vez» de los precios fijados en los proyectos de obras adjudicados antes del 1 de noviembre de 1956, en vir- 
tud del Decreto-ley de 18 de enero de 1957, para el Estado, y del Decreto de 22 de febrero de 1957, para las Corporaciones locales.

Los Decretos de 22 de mayo de 1963 y de 17 de octubre de 1963 volvieron a conceder, respectivamente, la actualización de los precios «por una sola vez» de contratas de obras licitadas antes de 1 de enero de 1963.

\section{Ley de Contratos del Estado.}

El 8 de abril de 1965 y por Decreto número 923 se aprobó el texto articulado de la Ley de Contratos del Estado. Su artículo 7. admite la posibilidad de inclusión de cláusulas de revisión de precios, las cuales se regularán por su legislación especial. Por otra parte, el artículo 46 establece que «la ejecución del contrato se realizará a riesgo y ventura del contratista y éste no tendrá derecho a indemnización por causa de pérdidas o averías o perjuicios causados en las obras sino en los casos de fuerza mayor. Para los efectos de esta Ley se considerarán como tales únicamente los que siguen:

1. Los incendios causados por electricidad atmosférica.

2. Los daños causados por terremotos y maremotos.

3. Los que provengan de los movimientos del terreno en que estén construidas las obras o que directamente las afecten.

4. Los detrozos ocasionados violentamente a mano armada en tiempo de guerra, sediciones populares o robos tumultuosos.

5. Inundaciones catastróficas, producidas como consecuencia del desbordamiento de ríos y arroyos, siempre que los daños no se hayan producido por la fragilidad de las defensas que hubiera debido construir el contratista en cumplimiento del contrato; y

6. Cualquier otro de efectos análogos a los anteriores, previo acuerdo del Consejo de Ministros».

7. DeCreto-ley 2/1964, De 4 DE FEbrero.

La legislación especial a que se refiere el artículo $7 .^{\circ}$ antes citado consiste en el Decreto-ley $2 / 1964$, de 4 de febrero, que regula las cláusulas de revisión de precios en los contratos del 
Estado y de los organismos autónomos. Se establece claramente que la inclusión de estas cláusulas es una potestad de la Administración, para los contratos de obras superiores a 5.000 .000 de pesetas y formalizados después del 4 de febrero de 1964. Se debe concertar para cada contrato mediante una fórmula polinómica, debiendo elaborarse fórmulas-tipo para cada clase de obra.

\section{SITUACIONES POSIBLES DE LOS CONTRATOS DE ENTES LOCALES}

Sobre este mosaico de disposiciones legales, trataremos de encajar las diversas situaciones que se pueden plantear a las Corporaciones locales.

\section{Contratos sin cláusula de revisión de precios.}

\section{A) Contratos en general.}

Se rigen, como hemos visto, por el Reglamento de Contratación de las Corporaciones locales, y supletoriamente (en virtud de su disposición adicional $2 .{ }^{\mathrm{a}}$ ), por toda la normativa aplicable al Estado sobre este mismo tipo de contratos.

El Reglamento de Contratación citado admite en su artículo 57-e) las revisiones de precios, de una manera automática, cuando se da la condición de una subida general de los precios, superior al 10 por 100 , ocasionada por un precepto obligatorio. Sin embargo, este apartado quedó suspendido por el Decreto de 25 de febrero de 1955, sin que hasta la fecha se haya derogado. Por lo tanto, a primera vista, parece que al no revisarse los precios ex lege ni haber cláusula de revisión expresa, habrá que atenerse a lo pactado, sin modificarlo por la subida del coste de los materiales o servicios empleados.

Sin embargo, la reciente sentencia del Tribunal Supremo de 15 de enero de 1971 admite la revisión de los precios de uno de estos contratos, señalando que el Decreto de 25 de febrero de 1955, que suspendía la aplicación del apartado e) citado, no era ya aplicable, apoyándose en los siguientes razonamientos: 
1.- Que el Decreto fue dictado con carácter temporal, ordenando no la derogación definitiva del repetido apartado, sino sólo su suspensión temporal, por lo que, al haber terminado la situación económica que determinó la promulgación de ese Decreto, debe entenderse derogado, y aplicable en cambio, por lo tanto, el apartado e) del artículo 57 del Reglamento de Contratación citado. Pero este razonamiento es, a nuestro juicio, inadmisible, porque, en realidad, lo que se ordenó con carácter temporal y transitorio fue la revisión automática de los precios (Ley de 17 de julio de 1945 y artículo 57-e) del Reglamento de Contratación, para el Estado y los entes locales, respectivamente), y la vuelta a la normalidad la constituye la suspensión de esas revisiones (por Decretos de 13 de enero de 1955 y de 25 de febrero siguiente). Así lo podemos ver en la Exposición de Motivos del citado Decreto de 13 de enero de 1955, que dice literalmente: «La Ley de 17 de julio de 1945 por la que se autorizó la revisión de los precios fijados en los proyectos de obras adjudicadas por subasta o concurso fue promulgada en atención a las fuertes alteraciones de los precios, consecuencia de la anormalidad económica a la sazón existente... Se observa al presente una regularidad en las licitaciones para la ejecución de las obras públicas, consecuencia de la normalidad económica conseguida, contrastada por las pequeñas o nulas variaciones de los índices que sirven de base a la revisión de los precios unitarios. Por ello, parece llegado el momento de usar de la facultad que la propia Ley reconoce de suspender la aplicación de sus preceptos, lo que permitirá, además, comprobar la efectiva superación de las dificultades que inspiraron aquella medida al propio tiempo que se ponderan los efectos del retorno a los principios que tradicionalmente han servido de base a la contratación administrativa».

Además, el artículo 5. del Código civil establece que «las leyes sólo se derogan por otras leyes posteriores, y no prevalecerá contra su observancia el desuso, ni la costumbre o práctica en contrario».

2. Que la temporalidad de este Decreto se comprobaba a la vista de las declaraciones reiteradamente hechas en toda esta 
legislación de que el régimen local en esta materia es paralelo al régimen estatal. Y como el sistema estatal tiene derogado el Decreto de 13 de enero de 1955, que ordenaba la suspensión de las revisiones de precios, por el nuevo Decreto 2/1964 y por la Ley de Contratos del Estado, que admiten estas revisiones, debe mantenerse ahora el paralelismo sosteniendo la vigencia del artículo 57-e) del Reglamento de Contratación de las Corporaciones locales. Ahora bien, examinemos ese paralelismo:

1) Revisiones automáticas (ex lege) para las subidas de precios debidas a aumentos establecidos por precepto obligatorio (para el Estado por la Ley de 1945 y para las Corporaciones locales por el artículo 57-e) del Reglamento de Contratación).

2) Suspensión de esas revisiones (Decretos de 13 de enero y de 25 de febrero de 1955, para el Estado y las Corporaciones locales, respectivamente).

3) Autorizaciones de revisiones ex lege por una sola vez (Decretos de 18 de enero y de 22 de febrero de 1957, y de 22 de mayo y 17 de octubre de 1963).

4) Revisiones reguladas detalladamente y condicionadas a la previa existencia de una cláusula de revisión. (Decreto de 4 de febrero de 1964 y artículo 7.. de la Ley de Contratos del Estado, que se aplican supletoriamente a las Entidades locales). Este sistema se refiere a supuestos diferentes de los de la legislación anterior, que se refería a todos los contratos y a las subidas de precios ocasionadas por preceptos obligatorios, puesto que sólo ampara a los contratos en que se haya pactado expresamente la revisión y extiende ésta a cualquier tipo de subida de precios. o sea, que las revisiones a que se refieren los apartados anteriores son las nacidas ex lege, que son independientes de las nacidas ex contractu, como señalaba ya la Ley de 1945 , y la regulación de éstas no supone la derogación de las normas aplicables a aquéllas.

Por todo ello no puede invocarse el paralelismo entre el Estado y las Corporaciones locales para demostrar la vigencia del artículo 57-e) del Reglamento de Contratación y la derogación del Decreto de 1955.

En este mismo sentido, la todavía más reciente sentencia del 
Tribunal Supremo de 5 de enero de 1972 afirma que «el invocado artículo quedó definitivamente suspendido por Decreto de 25 de febrero de 1955, y esta suspensión, pese a cuanto alega el recurrente, ya no fue levantada, porque las dos revisiones que autorizaron los Decretos de 22 de febrero de 1957 y 17 de octubre de 1963, lo fueron sobre contratos celebrados en ciertas épocas determinadas, que no alcanzan al ahora en cuestión, y además, con la expresión de que tales revisiones se hacían por una sola vez; por lo que al no existir disposición alguna que expresamente derogara la suspensión acordada por el Decreto de 25 de febrero de 1955, no cabe duda alguna de que la suspensión subsiste».

Y una vez desechada la aplicación del repetido artículo 57-e), ¿cuál es la normativa aplicable? La disposición adicional 2." del mismo Reglamento dice: «para lo no previsto en este Reglamento, regirán las disposiciones aplicables a la Administración general del Estado y, en su defecto, los preceptos pertinentes del Derecho privado». Estas disposiciones son las contenidas en la Ley de Contratos del Estado, que parte del principio de sujeción estricta a lo pactado, sin más excepciones que las que constituyen supuestos de fuerza mayor, enunciadas en el artículo 46 de dicha Ley, que es igual al artículo 57 del Reglamento de Contratación de las Corporaciones locales (una vez suprimido el apartado $e$ ), entre los cuales no está comprendida la elevación de los precios.

En resumen: en los contratos de las Corporaciones locales en que no haya cláusula de revisión expresa, no se podrán revisar los precios convenidos.

\section{B) Concesiones.}

El Reglamento de Servicios de las Corporaciones locales establece una regulación especial para las concesiones. En ellas la Administración puede modificar con toda libertad el servicio público que constituya su objeto, debiendo siempre compensar al concesionario por las modificaciones que le ordenare introducir.

En su artículo 126-2. dice: «En el régimen de concesión se diferenciará : 
a) el servicio objeto de la misma, cuyas características serán libremente modificables por el poder concedente y por motivos de interés público, y

b) la retribución económica del concesionario, cuyo equilibrio, a tenor de las bases que hubieran servido para su otorgamiento, deberá mantenerse en todo caso y en función de la necesaria amortización, durante el plazo de concesión, del coste de establecimiento del servicio que hubiere satisfecho, así como de los gastos de explotación y normal beneficio industrial».

¿Se puede entender que el párrafo citado establece un sistema especial de revisión de precios, en el que se pueda incluir como causa del mismo la elevación de los precios? Si consideramos que este artículo se refiere a la posibilidad de la Administración para modificar el servicio público, llegaremos a la conclusión de que el equilibrio económico que hay que mantener es el alterado por una modificación del objeto del contrato establecida unilateralmente por la Administración, pero no para asegurar al contratista contra toda eventualidad que le sobrevenga, pues esto sería una especie de seguro gratuito totalmente contrario al espíritu de los contratos, en que cada una de las partes debe correr con los riesgos que le sobrevengan, así como lucrarse con las circunstancias favorables.

Esta interpretación queda todavía aclarada con el artículo 127-2-2. del mismo Reglamento, que ordena a la Corporación concedente «mantener el equilibrio financiero de la concesión, para lo cual :

a) compensará económicamente al concesionario por razón de las modificaciones que le ordenare introducir en el servicio y que incrementaren los costos o disminuyeren la retribución, y

b) revisará las tarifas cuando, aun sin mediar modificaciones en el servicio, circunstancias sobrevenidas e imprevisibles determinaren, en cualquier sentido, la ruptura del equilibrio de la economía de la concesión».

Es decir, que la modificación de la retribución de un concesionario puede conseguirse porque la Administración haya ordenado modificar la prestación, o bien porque aun sin modificar esta prestación, hayan sobrevenido circunstancias imprevisibles. 
Sin embargo, ¿cabe encajar la alteración de los precios entre esas circunstancias imprevisibles? La jurisprudencia ha aclarado que «el aumento del jornal de los peones en la industria de la construcción no puede calificarse de caso fortuito, por faltarle el primer requisito esencial, o sea, el no estar sujeto a la previsión humana, o que, estándolo, haya imposibilidad de evitarlo, ya que en la fecha en que se contrajo la obligación, dada la inestabilidad de los medios económicos no podía escapar tal circunstancia a la previsión de los contratantes» (sentencia de $18 \mathrm{de}$ junio de 1947). «Si bien esta Sala tiene declarada la aplicabilidad de aquella cláusula-cláusula rebus sic stantibus-, no es menos cierto que en el caso del pleito, ni la elevación de los jornales que se invoca llegó a ser extraordinaria como para relevar al contratista de cumplir, ni cae dentro de lo totalmente imprevisible, supuestos imprescindibles para que pudiera entrar en juego la referida cláusula, tanto más cuanto que los contratos de ejecución de obras por el precio de adjudicación de la subasta se celebran a riesgo y ventura y debe en ellos, por regla general, pechar el contratista con los aumentos que se produzcan en los jornales o en los materiales» (sentencia de 20 de junio de 1958, y en el mismo sentido, la sentencia de 4 de julio de 1951).

Por todo ello, se llega a la conclusión de que la retribución a los concesionarios no debe revisarse por subida de precios, a no ser que se demuestre que no ha podido preverse tal subida, y siempre, salvo pacto expreso en contrario.

\section{Contratos con cláusula de revisión de precios.}

Los Reglamentos de Contratación y de Servicios de las Corporaciones locales no hacen ninguna mención a las cláusulas de revisión de precios. Por lo tanto regirán, tal como ordenan respectivamente sus disposiciones adicional 2. ${ }^{a}$ y final 2. mas aplicables a la Administración general del Estado y, en su defecto, los preceptos pertinentes del Derecho privado.

Por ello habrá que acudir al artículo 1.255 del Código civil, que autoriza todo tipo de cláusulas en los contratos, y al artículo 10 de la Ley de Contratos del Estado, que señala expresa- 
mente que «la Administración puede concertar con los particulares los pactos y condiciones que tenga por convenientes, siempre que no sean contrarios al interés público, al ordenamiento jurídico o a los principios de la buena administración».

Es decir, que las cláusulas de revisión de precios, como cualquier otra cláusula lícita, están permitidas en los contratos que celebren las Corporaciones locales. Además, uno de los principios generales del Derecho es el de la libertad de contratación, que presume la posibilidad de cualquier pacto, mientras no esté expresamente prohibido.

Ahora bien, el artículo $7 .^{\circ}$ de la citada Ley establece que «la inclusión de cláusulas de revisión de precios se regulará por su legislación especial». Esta regulación especial se reduce al Decreto-ley 2/1964, de 4 de febrero, desarrollado por el Decreto $401 / 1971$, de 11 de marzo, los cuales se refieren a los contratos de obras.

\section{A) Contratos de obras.}

a) Los contratos de obras a que se refieren los citados Decretos son los de importe superior a $\mathbf{5 . 0 0 0 . 0 0 0}$ de pesetas formalizados después del 6 de febrero de 1964 (fecha en que se promulgó el Decreto-ley 2/1964).

Para estos contratos se preceptúa que las cláusulas de revisión de precios que contengan consistirán en una fórmula polinómica compuesta «por varios sumandos que se obtendrán multiplicando los tantos por uno de los elementos básicos que integran la obra por la relación entre sus respectivos precios en la fecha en que se aplique la revisión y la fecha de licitación; se completarán con un sumando fijo, cuyo valor será el tanto por uno correspondiente a los gastos que han de permanecer invariables, como son la amortización e interés de las inversiones en maquinaria y medios auxiliares, el beneficio previsto y los costes correspondientes a elementos no básicos. Este sumando fijo no podrá ser superior a 0,15 , y la suma de todos los sumandos en cada fórmula será igual a uno». Además se prevé que para cada. tipo de contrato se redactará una fórmula-tipo por cada Departamento ministerial. (Recientemente, por Decreto de 19 de di- 
ciembre de 1970, se han redactado, para el año 1971, una serie de fórmulas-tipo aplicable a todos los Departamentos ministeriales, y derogando las antiguas fórmulas, relacionadas en el mismo Decreto, que se habían ido dictando por separado para cada Ministerio).

En cuanto a los precios que servirán de comparación para ver su incremento en cada factor de la producción, serán los que señale mensualmente el Comité Superior de Precios de Contratos del Estado, que se publican en el Boletín Oficial del Estado.

Como requisitos para la apertura de la revisión están:

1. ${ }^{-}$El de no haber incurrido en mora el contratista (recordemos que en los contratos administrativos se incurre en mora automáticamente al rebasar los plazos de ejecución convenidos);

$2 .^{\circ}$ El de haber certificado ya por lo menos el 20 por 100 de la obra (este 20 por 100 no es susceptible de revisión), y

3. - El de que la subida de los precios resultante de los indices oficiales de precios sea superior a un 15 por 100 del coste inicial.

Además, del coeficiente de revisión que resulte habrá que deducir un 2,5 por 100 , que correrá a cargo del contratista por considerarse que es el riesgo ordinario de subida de precios.

Toda esta normativa, como hemos visto, es aplicable a los contratos de las Corporaciones locales. Ahora bien, ¿qué sucede cuando se ha pactado por una Corporación una cláusula de revisión de precios que no se sujeta a estas reglas? A nuestro juicio se trata de un tipo de cláusulas de contenido normado, en las que se determina por la Ley el alcance de los derechos y obligaciones de cada uno de los contratantes. En estos contratos normados o reglamentados, como señala Hernández Gil, «cabe contratar o no; cabe hacerlo con una persona o con otra. Mas si el contrato se celebra, la fijación de su contenido no se entrega totalmente a la libre determinación de las partes, sino que lo predetermina la norma»... «En estos casos el contrato representa el acto de voluntad por el que las personas que lo conciertan se sitúan en la zona de actuación de la norma».

Es decir, que los pactos distintos establecidos por las Corporaciones locales serán nulos por ser contrarios a la Ley. Sin em- 
bargo, atendiendo a la naturaleza del negocio y a la intención de las partes, no deberá negarse la revisión de precios, sino que deberá admitirse, pero siempre sujetándose a los preceptos legales vigentes. El rechazar una forma de revisión no supone el negar la posibilidad de la revisión, sino sólo el aplicar la forma de revisión que la Ley ordena, prescindiendo de los pactos contrarios a la misma.

b) En cuanto a los contratos anteriores al Decreto-ley 2/1964, tal como dispone su disposición transitoria $1 . \stackrel{\mathrm{a}}{\text {, si }}$ son superiores a 5.000 .000 de pesetas, se les aplicará la revisión en los términos del Decreto-ley, a petición de los contratistas (aun sin cláusula), y si son inferiores a dicha cantidad les será de integra aplicación el Decreto-ley de 10 de octubre de 1963, que admitía las revisiones con una gran generosidad para los contratistas.

c) $\mathrm{Y}$ respecto a los contratos de obras posteriores al Decreto-ley 2/1964, pero inferiores a 5.000.000 de pesetas, dada la redacción del mismo, parece que no se admite la posibilidad de inclusión de ninguna cláusula de revisión de precios.

\section{B) Contratos de servicios y suministros.}

Para ellos no hay ninguna legislación especial sobre cláusulas de revisión de precios. Por lo tanto, el primer problema que se plantea es el de la posibilidad de existencia de las mismas.

Como hemos visto, en ninguna parte aparece expresamente una autorización para su inclusión, pero tampoco hay ninguna prohibición, por lo que habrá que entender, como expusimos antes, que es posible incluirlas. También ofrece base para ello el artículo 7. de la Ley de Contratos del Estado, que autoriza la inclusión de las mismas, y que está comprendido dentro del título preliminar de la misma, que se refiere tanto a obras como a contratos de servicios y suministros. Es decir, que la Ley prevé la existencia de estas cláusulas para los tres tipos de contratos: y no sólo para el de obras.

Ante la ausencia de «legislación especial» que regule la materia, caben dos posibles soluciones:

1. Aplicar supletoriamente a estos contratos lo establecido 
para los contratos de obras por el Decreto-ley 2/1964, en atención a que los artículos 67 y 84 de la Ley de Contratos del Estado y 208 y 238 de su Reglamento disponen que los contratos de servicios y de suministros, respectivamente, se regirán por las disposiciones correspondientes a los contratos de obras.

2. Estimar, ante la ausencia de normas específicas, que las partes tienen completa libertad para contratar y para ejecutar lo contratado, sin más límites que los establecidos en general para todo tipo de contratos administrativos, es decir, que no sean contrarios al interés público ni al ordenamiento jurídico.

La solución más correcta parece la segunda, y ello por las siguientes razones:

- La remisión de los contratos de servicios y suministros a los de obras no se hace, en general, para toda normativa, sino sólo para el título I de la Ley y título II de su Reglamento, entre los cuales no se encuentra la regulación de las cláusulas de revisión, ni siquiera su remisión a la «legislación especial». Es decir, que la Ley de Contratos del Estado prevé estas cláusulas para todo tipo de contratos, y se remite a su legislación especial, aun cuando dicha legislación se refiera sólo a los contratos de obras, sin que, estrictamente, haya nada que disponga su aplicación supletoria a los contratos de servicios y suministros.

- La naturaleza de los contratos referidos admite, en cualquiera de ellos, la inclusión de una cláusula de revisión de precios, porque se trata de situaciones en las que la ejecución de lo contratado puede diferirse varios años desde el momento de fijación de los precios. Sin embargo, el contenido de todos estos contratos es muy diferente, y por ello malamente serán aplicables a unos las reglas de los otros.

- No habrá ninguna fórmula-tipo aplicable a estos contratos, pues todas las que se han publicado lo han sido en ejecución del Decreto-ley 2/1964 y se refieren, por lo tanto, solamente a diferentes clases de obras, pero no a servicios ni suministros.

\section{CRITICA DEL SISTEMA}

La principal objeción que se puede hacer al referido sistema es la de su casuismo, su falta de generalidad. 
En primer lugar aparece una discriminación injustificada entre los contratos realizados por la Administración y el resto de los contratos realizados por los particulares. ¿Por qué razón va a conceder la Administración a sus contratistas unas ventajas que los demás empresarios no conceden? Este problema se agudiza todavía más en aquellos sistemas como el establecido por la Ley de 1945 o el transitorio del Decreto-ley de 10 de octubre de 1963, en los cuales se concedía siempre la revisión a los contratos realizados por la Administración. Entre éstos se puede incluir también el sistema que algunos consideran vigente para las Corporaciones locales al amparo del artículo 57-e) de su Reglamento de Contratación.

Se pretendió justificar esta diferencia entre la Administración y los particulares apoyándose en la teoría del factum principis o «hecho del príncipe», según la cual el Estado es el causante de la elevación de los precios de determinados bienes o servicios, porque asi lo ha establecido en un precepto obligatorio. Por lo tanto, tiene que someterse él mismo a sus propios actos y aplicar esa elevación en sus contratos. Sin embargo, a esta teoría se le pueden hacer varias objeciones:

1. - En todo caso, el responsable de la elevación de los precios será el Estado, pero no las demás entidades que también tienen la consideración de Administración pública. $Y$ así, por ejemplo, no se puede decir que un Ayuntamiento sea causante de la elevación en todo el territorio nacional del salario mínimo. Por ello el único ente de Derecho público para quien se podría invocar dicha teoría sería el Estado, pero no las Entidades locales ni los organismos autónomos.

2. El Estado, según esta teoría, tendría que hacerse responsable de las elevaciones de los precios establecidas por precepto obligatorio, pero no para las producidas espontáneamente en un mercado de libre competencia, pues, aunque es cierto que indirectamente es causante de ellas por haber organizado la vida económica de la Nación, también es cierto que los demás miembros de la misma participan activamente en esta vida económica y son también responsables, en mayor o menor parte, junto con los demás factores que intervienen en la determinación de la 
oferta y la demanda, del nivel que alcancen los precios de cada bien del mercado.

3. $\quad$ Aunque el Estado sea responsable de las elevaciones de los precios, esto no justifica que revise los precios de sus anteriores contratos, pues si bien es cierto que debe ajustarse a sus propios preceptos, también es cierto que los particulares están igualmente sometidos a ellos. Es decir, si el cumplimiento de un decreto de elevación del salario mínimo no significa necesariamente la revisión del precio de una obra para un particular, tampoco lo significará para el Estado.

Otra «discriminación» criticable es la de diferenciar el tratamiento de los contratos con cláusula de revisión de los que no la tengan. Desde luego es un mal menor en comparación con la revisión automática ex lege de todos los contratos en que intervenga la Administración, porque esto supondría un auténtico privilegio de los contratistas de la Administración en perjuicio de los demás contratistas. Pero por otra parte se deja a la Administración una nueva potestad discrecional, la de incluir o no en cada contrato la cláusula de revisión, de la que se puede hacer un uso arbitrario e injusto.

El que la Administración revise los precios de sus contratos y no deje hacerlo a los particulares, poniendo trabas a las cláusulas de revisión en los contratos privados alegando que agudizan todavía más el peligroso proceso inflacionista, llegaría a ser una auténtica competencia desleal para éstos.

$Y$, enfocándolo desde otro punto de vista, si en el mercado privado se realizan los contratos sin revisión de los precios, la Administración podrá también contratar sus necesidades sin tener que hacer esas revisiones a costa de los fondos públicos, que supondrían una auténtica mala administración de los mismos.

\section{CONCLUSIONES PRACTICAS}

1. Contratos sin cláusula de revisión de precios:

Contratos en general: No cabe revisión (Decreto de 25 de febrero de 1955, derogatorio del artículo 57-e) del Reglamento de Contratación). 
Concesiones: No hay revisión, salvo que se demuestre que no pudo preverse la subida de los precios (artículos 126-2 y 127-2 del Reglamento de Servicios).

2. Contratos con cláusula de revisión de precios:

Contratos de obras:

Superiores a 5.000.000 de pesetas:

Formalizados antes del 6 de febrero de 1964: Revisión a petición del contratista, aun sin cláusula, en los términos del Decreto-ley 2/1964 (disposición transitoria 1. ${ }^{\mathrm{a}}$ del mismo).

Formalizados después del 6 de febrero de 1964: Revisión conforme a las normas del Decreto-ley 2/1964.

Inferiores a 5.000 .000 de pesetas:

Formalizados antes del 6 de febrero de 1964: Aplicación del Decreto-ley de 10 de octubre de 1963 (disposición transitoria 1." del Decreto-ley 4/1964).

Formalizados después del 6 de febrero de 1964: Sin revisión, por no existir posibilidad de incluir esta cláusula.

Contratos de servicios y suministros: Sujeción a lo pactado. 
REVL-1972, núm. 175. ANGULO GONZALEZ, MARIA ANTONIA. REVISIONES DE PRECIOS EN LO... REVL-1972, núm. 175. ANGULO GONZALEZ, MARIA ANTONIA. REVISIONES DE PRECIOS EN LO... 